\title{
Changes in salivary physiological stress markers associated with winning and losing
}

\author{
Masako Hasegawa, Masahiro Toda and Kanehisa Morimoto \\ Department of Social and Environmental Medicine, Osaka University Graduate School of Medicine, Osaka, Japan \\ (Received 24 October 2007; and accepted 21 November 2007)
}

\begin{abstract}
Using a representative table game popular in Japan known as shogi, or Japanese chess, we investigated the effects of winning and losing on saliva composition. The subjects were 90 healthy male university students who were members of a shogi club. Saliva samples were collected immediately before and after playing shogi, and again $30 \mathrm{~min}$ later. Salivary cortisol and testosterone levels in the samples were determined by ELISA and EIA, respectively. After finishing each game, the competitiveness of the game was evaluated using questionnaires. In the samples taken after playing shogi, there was an increase in the levels of salivary testosterone and cortisol, regardless of whether the subject won or lost, and the tendency was more pronounced in competitive games. There were no such changes in the control group, who did not play a game prior to providing the samples. Our results suggest that stress response is intimately linked with competition and could be used to determine which players are more capable of handing stress in a competitive environment.
\end{abstract}

There are a number of confrontational situations that occur in nature. Of those, competition involves two or more individuals struggling for mastery while attempting to achieve the same goal. Thus, along with winners there are also losers. Previous research indicates that sports and gambling participation induce stress $(3,14)$. However, those studies did not investigate the interactions between winners and losers, and how competitive stress triggers physiological symptoms has not been revealed.

McCaul (13) reported greater increases of testosterone in saliva and more positive moods in winners than losers among individuals who participated in a task entirely controlled by chance (coin tossing). In another experiment (11), subjects were awarded

Address correspondence to: Prof. Kanehisa Morimoto Department of Social and Environmental Medicine, Osaka University Graduate School of Medicine, 2-2 Yamada-Oka, Suita, Osaka 565-0871, Japan Tel: +81-6-6879-3920, Fax: +81-6-6879-3929

E-mail: morimoto@envi.med.osaka-u.ac.jp
$\$ 100$ prizes depending on a random lottery. Winners in those situations, who won without any effort of their own, did not show subsequent testosterone increases in blood that were greater than those of the losers. These experiments, conducted in a casual manner, do not necessarily indicate that the derived conclusion reflects stress reaction.

Real competition, especially related to sports, has been well researched in psychoneurotic endocrinological studies, though universal agreement on a number of factors has not been reached. For example, one study reported that levels of testosterone and cortisol in blood samples from winning wrestlers rose higher above the level of the previous match as compared to the losing wrestlers (15), whereas another study reported that those levels were not different between winners and losers (5). The reason for this discrepancy may be related to physical stress caused by participation in sporting events. In fact, those stress measurements may have been affected by psychological stress induced by competition. 
In the present investigation, we studied subjects who participated in a table game popular in Japan known as shogi, or Japanese chess, in order to exclude the effects of physical stress from the environment of competition, and measured the levels of testosterone and cortisol in saliva samples. The competitiveness of shogi is partly derived from its comparatively long period of playing, thus we considered it an effective means to study psychological stress. In addition, we reviewed the competitive aspects of each game, to determine the intensity of the competition.

\section{MATERIALS AND METHODS}

The subjects were 90 healthy male students, who were members of the shogi club at Osaka University and participating in the Western Japan Convention hosted by the Kansai Student Shogi Association. None were smokers or taking any medications. Forty one of the subjects (mean $\pm \mathrm{SD}, 21.3 \pm 2.7$ years old) played shogi during the convention (shogi group) and $49(19.5 \pm 2.0$ years old) watched the games (control group). All subjects gave consent to participate in the study, after being informed of its purpose and method. Saliva samples were collected from both groups immediately before and after the games were played, and again 30 min later (Fig. 1) using a 50-mL polypropylene conical tube (BLUE $\left.\mathrm{MAX}^{\mathrm{TM}}\right)$. The subjects were requested to refrain from eating and drinking at least $2 \mathrm{~h}$ before the first sampling (19). The samples were stored at $-30^{\circ} \mathrm{C}$ until the assay. Salivary cortisol levels were determined by ELISA (enzyme-linked immunosorbent assay) and testosterone levels by EIA (enzyme immunoassay), using previously described methods ( 7 , $8,18)$. Following each game, the shogi group reported whether the game was competitive or noncompetitive. Values were converted to percentages of the baseline (before playing shogi). ANOVA with repeated measures was performed to examine time-

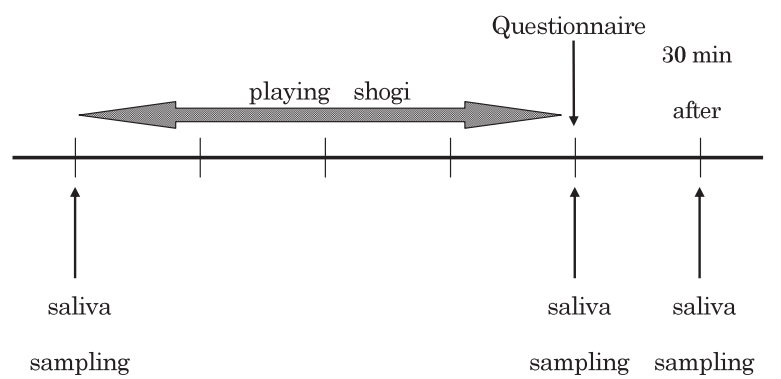

Fig. 1 Experimental protocol related differences and Bonferroni's test was used for multiple comparisons. Values were considered to be significantly different at $p<0.05$.

\section{RESULTS}

In samples from the shogi group taken after playing the game, there were significant increases in the levels of salivary testosterone $(63.8 \pm 21.7$ to $73.2 \pm$ $20.9 \mathrm{pg} / \mathrm{mL} ; p<0.01)$ and cortisol $(0.446 \pm 0.281$ to $0.800 \pm 0.354 \mu \mathrm{g} / \mathrm{dL} ; p<0.05$ ) (Fig. 2), though the increased levels were not maintained in the samples taken $30 \mathrm{~min}$ later (testosterone and cortisol levels were $63.7 \pm 17.2 \mathrm{pg} / \mathrm{mL}$ and $0.473 \pm 0.251 \mu \mathrm{g} / \mathrm{dL}$, respectively). As for the control group, there were no significant changes in the levels of cortisol and testosterone. We categorized the samples taken from the shogi group into 2 sub-groups, winners and losers, based on the outcome of the game, however, found was no significant difference in salivary tes-
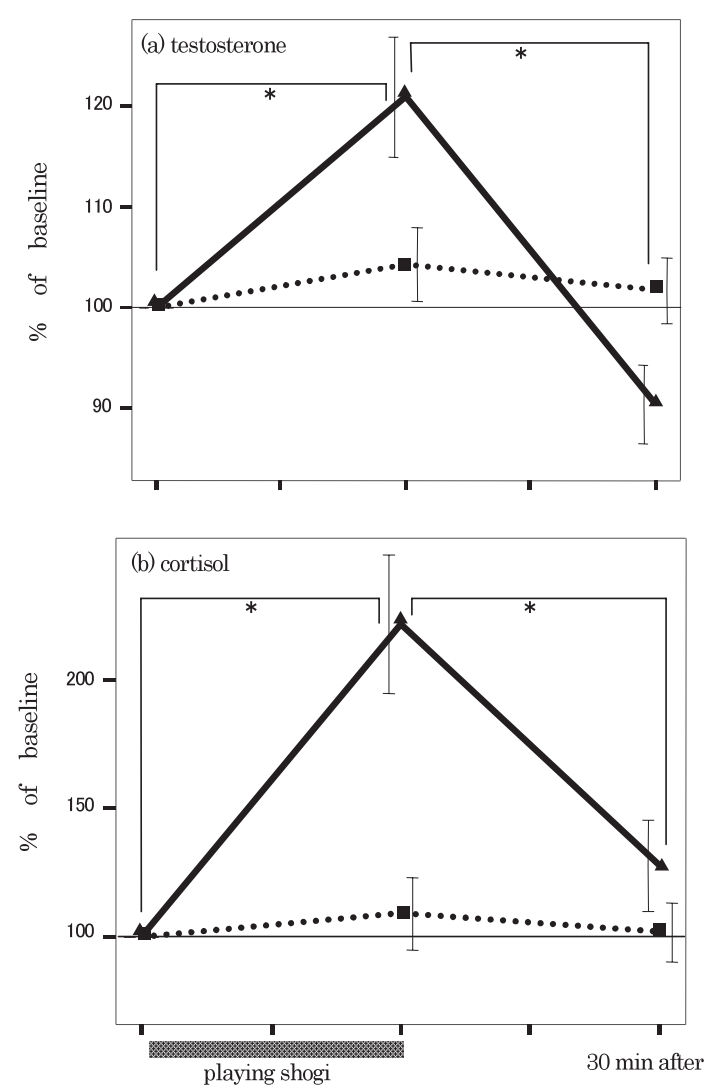

Fig. 2 Mean values $( \pm \mathrm{SE})$ are presented as percentages of the baseline values obtained before playing a game. (a) Salivary testosterone and (b) cortisol in samples taken from the shogi $(\boldsymbol{\Delta}, n=41)$ and control $(\boldsymbol{\square}, \mathrm{n}=49)$ groups. ${ }^{*}$ Significantly different $(p<0.05)$ as determined by repeated measures ANOVA and Bonferonni's test. 
tosterone level between the sub-groups (Fig. 3). However, the increase seen in the winners had a relatively greater amount of reduction in the samples taken $30 \mathrm{~min}$ later. Salivary cortisol showed the same tendency, and the difference was not significant. We also categorized the samples taken from the shogi group into 2 sub-groups, competitive and non-competitive, according to the questionnaire results. In the samples taken following a competitive game, there were significant increases in the levels of salivary testosterone $(66.0 \pm 23.9$ to $76.8 \pm$ $20.7 \mathrm{pg} / \mathrm{mL} ; p<0.01)$ and cortisol $(0.477 \pm 0.305$ to $0.880 \pm 0.380 \mu \mathrm{g} / \mathrm{dL} ; p<0.05$ ) (Fig. 4), whereas there were no such changes in the samples taken following a non-competitive game.

\section{DISCUSSION}

Rising levels of salivary testosterone and cortisol were identifiable in samples taken from the shogi
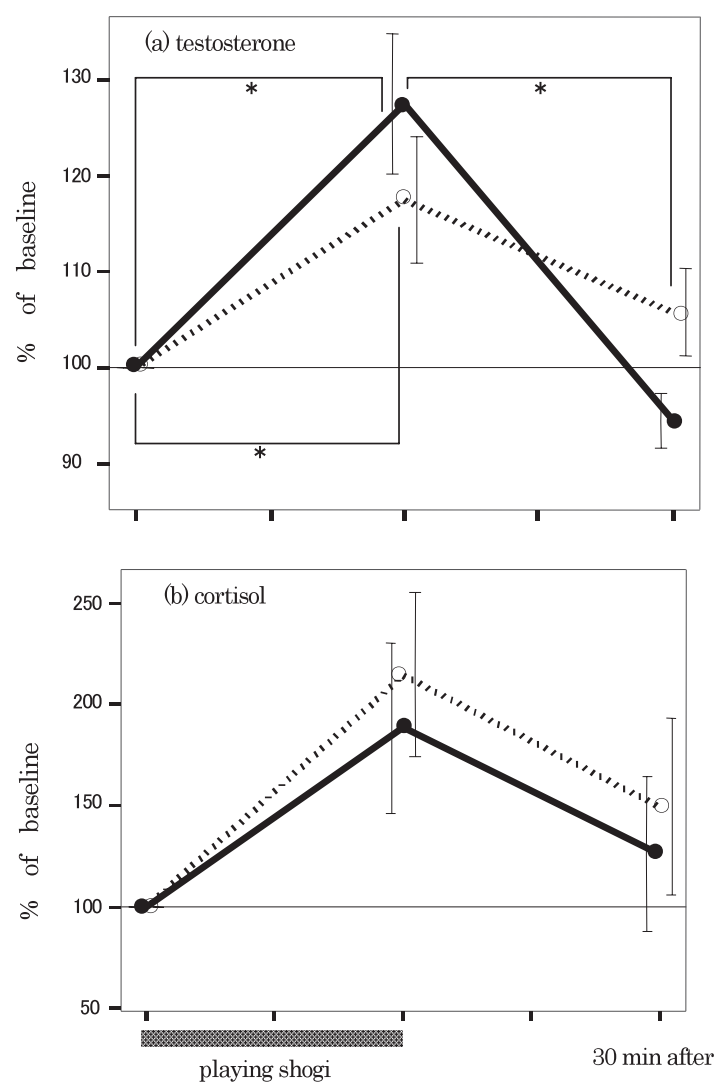

Fig. 3 Mean values $( \pm$ SE) are presented as percentages of the baseline values obtained before playing a game. (a) Salivary testosterone and (b) cortisol levels were measured in the winners $(0, n=15)$ and losers $(\bigcirc, n=26)$. *Significantly different $(p<0.05)$, as determined by repeated measures ANOVA and Bonferonni's test. group, whereas those from the control group were considered to reveal no effect from the game on the endocrine system. It has been reported that the perceptions of winning and losing regardless of actual performance or merit had different influences on testosterone levels. In a previous study, testosterone levels showed increases and decreases in fans, depending on the outcome of their team (2). For this reason, we expected that the levels of testosterone in samples from the control group would change, however, we were not able to confirm such results. We attributed this to the fact that the present control group differed in a number of ways from fans cheering for their team.

As for the participants, Mazur et al. (12) compared samples taken following competitive and noncompetitive chess games, and found that the levels of salivary testosterone were significantly different. Our results (Fig. 4) confirmed those and suggested that stress response is intimately linked with com-
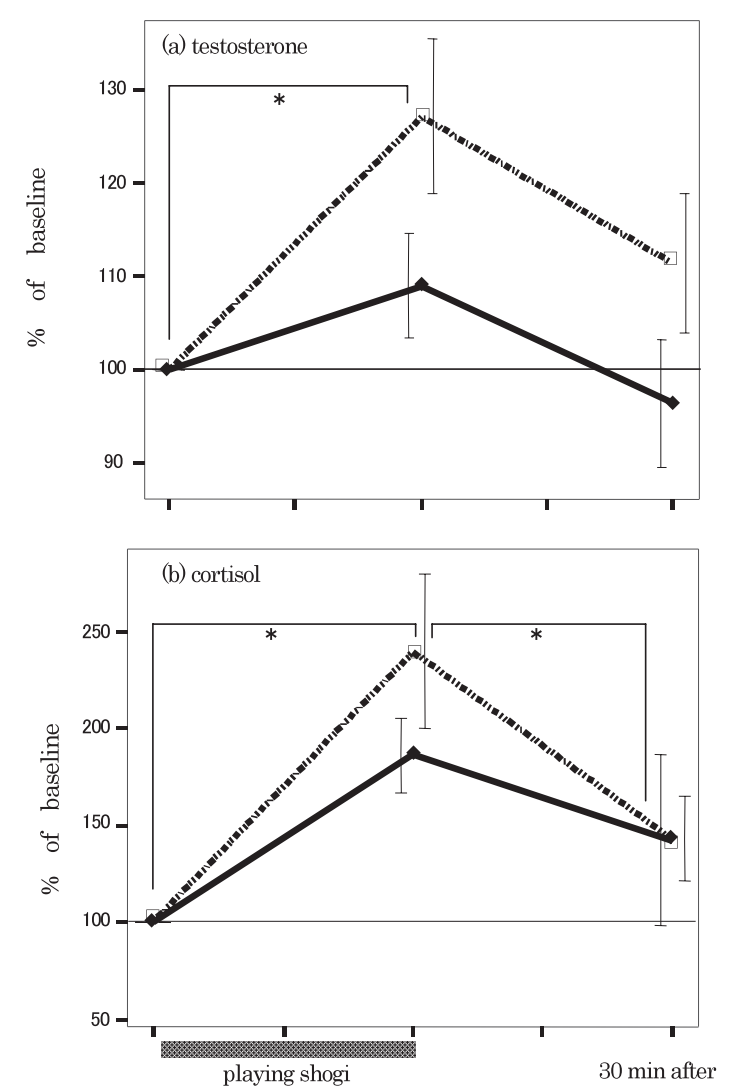

Fig. 4 Mean values $( \pm$ SE) are presented as percentages of the baseline values obtained before playing a game. (a) Salivary testosterone and (b) cortisol levels in samples taken following non-competitive $(\checkmark, n=14)$ and competitive $(\square$, $\mathrm{n}=27$ ) games. *Significantly different $(p<0.05)$, as determined by repeated measures ANOVA and Bonferonni's test. 
petitiveness, based on our assumption that the sense of stress following a competitive game would continue 30 min later. On the other hand, in a non-competitive game, players are able to anticipate the result before the game is finished, resulting in a lower sense of stress, because of feeling of resignation from defeat or relief from victory toward the end of the game. Thus, the stress responses of participants are inhibited during a non-competitive game.

In the shogi group, there were no differences in levels of salivary testosterone and cortisol between the winners and losers. This supports a previous report (16), which found that there were no differences in samples collected from male judo wrestlers in regard to the levels of testosterone and cortisol in blood taken before and after a match. Some studies have suggested that testosterone levels are related to positive coping behavior $(9,10)$. That is, positive coping behavior in individuals sensing stress causes elevated levels of testosterone and activity of the sympathetic nervous system. In addition, when an individual has self-confidence and experiences positive emotions, they tend to view stressful situations as challenging $(2,4,6)$. Therefore, in the present study, it is possible that the winners demonstrated positive coping behavior and the losers negative coping behavior. It is important for future studies to examine the relationships among the endocrine system and a number of factors that influence stress response, such as lifestyle, personality, and stress coping behavior. Additionally, because the mechanism for such a testosterone effect is unclear even in non-human animals (17), we intend to reveal it in future studies.

\section{REFERENCES}

1. Bandura A (1991) Self-efficacy mechanism in physiological activation and health promoting behavior. In: Neurobiology of Learning, Emotion, and Affect (Mudden J, ed.), pp229269, Raven Press, New York.

2. Bernhardt PC, Dabbs Jr. JM, Fielden JA and Lutter CD (1998) Testosterone changes during vicarious experiences of winning and losing among fans at sporting events. Physiol Behav 65, 59-62.

3. Chatterton Jr. RT, Vogelsong KM, Lu YC and Hudgens GA (1997) Hormonal responses to psychological stress in men preparing for skydiving. $J$ Clin Endocrinol Metab 82, 2503 2509.

4. Dienstbier RA (1989) Arousal and physiological toughness: implications for mental and physical health. Psychol Rev 96, 84-100.

5. Elias M (1981) Serum cortisol, testosterone, and testosteronebinding globulin responses to competitive fighting in human males. Aggressive Behav 7, 215-224.

6. Eubank M, Collins D, Lovell G, Dorling D and Talbot S (1997) Individual temporal differences in precompetition anxiety and hormonal concentration. Person Individ Diff 23, 1031-1039.

7. Granger DA, Schwartz EB, Booth A and Arentz M (1999) Salivary testosterone determination in studies of child health and development. Horm Behav 35, 18-27.

8. Granger DA, Shirtcliff EA, Booth A, Kivlighan KT and Schwartz EB (2004) The "trouble" with salivary testosterone. Psychoneuroendocrinology 29, 1229-1240.

9. Henry JP (1986) Neuroendocrine patterns of emotional response. In: Emotion: Theory, Research and Experiences (Plutchik R and Kellerman H, eds.), pp37-60, Academic Press, San Diego.

10. Koolhaas JM, Korte SM, De Boer SF, Van Der Vegt BJ, Van Reenen CG, Hopster H, De Jong IC, Ruis MA and Blokhuis HJ (1999) Coping styles in animals: current status in behavior and stress-physiology. Neurosci Biobehav Rev 23, 925935.

11. Mazur A and Lamb TA (1980) Testosterone, status and mood in human males. Horm Behav 14, 236-246.

12. Mazur A, Booth A and Dabbs JM (1992) Testosterone and chess competition. Social Psychol 55, 70-77.

13. McCaul KD, Gladue BA and Joppa M (1992) Winning, losing, mood, and testosterone. Horm Behav 26, 486-504.

14. Meyer G, Hauffa BP, Schedlowski M, Pawlak C, Stadler MA and Exton MS (2000) Casino gambling increases heart rate and salivary cortisol in regular gamblers. Biol Psychiatry 48, 948-953.

15. Passelergue P and Lac G (1999) Saliva cortisol, testosterone and $\mathrm{T} / \mathrm{C}$ ratio variations during a wrestling competition and during the post-competition recovery period. Int $J$ Sports Med 20, 109-113.

16. Salvador A, Simon V, Suay F and Llorens L (1987) Testosterone and cortisol responses to competitive fighting in human males: A pilot study. Aggressive Behav 13, 9-13.

17. van Anders SM and Watson NV (2007) Effects of abilityand chance-determined competition outcome on testosterone. Physiology Behavior 90, 634-642.

18. Shimada M, Takahashi K, Ohkawa T, Segawa M and Higurashi M (1995) Determination of salivary cortisol by ELISA and its application to the assessment of the circadian rhythm in children. Horm Res 44, 213-217.

19. Toda M, Morimoto K, Nagasawa S and Kitamura K (2004) Effect of snack eating on sensitive salivary stress markers cortisol and chromogranin A. Environ Health Prev Med 9, 27-29. 